\title{
BMJ Open MRI for measuring therapy efficiency after revascularisation in ST-segment elevation myocardial infarction: a systematic review and meta-regression analysis
}

\author{
Benjamin Kendziora (D , ${ }^{1}$ Heli Stier, ${ }^{1}$ Peter Schlattmann, ${ }^{2}$ Marc Dewey ${ }^{1}$
}

To cite: Kendziora B, Stier H, Schlattmann P, et al. MRI for measuring therapy efficiency after revascularisation in STsegment elevation myocardial infarction: a systematic review and meta-regression analysis. BMJ Open 2020;10:e034359. doi:10.1136/ bmjopen-2019-034359

- Prepublication history and supplemental material for this paper are available online. To view these files, please visit the journal online (http://dx.doi. org/10.1136/bmjopen-2019034359).

Received 16 September 2019 Revised 19 June 2020 Accepted 30 July 2020

Check for updates

(C) Author(s) (or their employer(s)) 2020. Re-use permitted under CC BY-NC. No commercial re-use. See rights and permissions. Published by BMJ.

${ }^{1}$ Institute of Radiology, Charité - Universitätsmedizin Berlin, Humboldt-Universität and Freie Universität, Berlin, Germany

${ }^{2}$ Institute of Medical

Statistics, Computer

Sciences and Documentation,

Universitätsklinikum Jena,

Friedrich-Schiller-Universität, Jena, Germany

Correspondence to

Dr Marc Dewey;

marc.dewey@charite.de

\section{ABSTRACT}

Objective To summarise existing data on the relation between the time from symptom onset until revascularisation (time to reperfusion) and the myocardial salvage index (MSI) calculated as proportion of nonnecrotic myocardium inside oedematous myocardium on T2-weighted and T1-weighted late gadolinium enhancement MRI after ST-segment elevation myocardial infarction (STEMI).

Methods Studies including patients with revascularised STEMI and stating both the time to reperfusion and the MSI measured by T2-weighted and T1-weighted late gadolinium enhancement MRI were searched in MEDLINE, EMBASE and ISI Web of Science until 16 May 2020. A mixed effects model was used to evaluate the relation between the time to reperfusion and the MSI. The gender distribution and mean age in included patient groups, the timing of MRI, used MRI sequences and image interpretation methodology were included in the mixed effects model to explore between-study heterogeneity.

Results We included 38 studies with 5106 patients. The pooled MSI was $42.6 \%$ (95\% Cl: 38.1 to 47.1$)$. The pooled time to reperfusion was 3.8 hours ( $95 \% \mathrm{Cl}$ : 3.5 to 4.0). Every hour of delay in reperfusion was associated with an absolute decrease of $13.1 \%(95 \% \mathrm{Cl}$ : 11.5 to $14.6 ; p<0.001)$ in the MSI. Between-study heterogeneity was considerable $\left(\sigma^{2}=167.8\right)$. Differences in the gender distribution, timing of MRI and image interpretation among studies explained $45.2 \%$ of the between-study heterogeneity.

Conclusions The MSI on T2-weighted and T1-weighted late gadolinium enhancement MRI correlates inversely with the time to reperfusion, which indicates that cardioprotection achieved by minimising the time to reperfusion leads to a higher MSI. The analysis revealed considerable heterogeneity between studies. The heterogeneity could partly be explained by differences in the gender distribution, timing and interpretation of MRI suggesting that the MRI-assessed MSI is not only influenced by cardioprotective therapy but also by patient characteristics and MRI parameters.
Strengths and limitations of this study

- A comprehensive search in three electronic databases was performed and a large number of studies could be included.

State-of-the-art statistical tests for meta-regression analyses were applied.

- Data on T2-weighted and T1-weighted mapping MRI, which is a valuable and increasingly used alternative to conventional T2-weighted and T1-weighted late gadolinium enhancement MRI for quantifying oedematous and fibrotic myocardium, are not included in the meta-regression analysis.

\section{INTRODUCTION}

Despite a rising incidence of myocardial infarction worldwide, a reduction in mortality has been observed in industrialised nations. ${ }^{1}$ This reduction is attributable to salvage of myocardium by therapeutic reopening of culprit arteries using either percutaneous coronary intervention or fibrinolysis. ${ }^{2}$ Salvaged myocardium is defined as the difference in size between the previously ischaemic area at risk distal to the obstructed coronary artery and the final infarct size. ${ }^{3}$ Quantification of salvaged myocardium after revascularisation therapy allows evaluation of therapeutic efficiency and can be used as an outcome parameter in studies that investigate cardioprotective strategies. ${ }^{3}{ }^{99 \mathrm{~m}}$ Technetiumsestamibi single-photon emission tomography (SPECT) is currently the reference standard for quantification of salvaged myocardium. ${ }^{3}$ Unfortunately, there are several disadvantages that limit its use. SPECT involves radiation exposure and is logistically demanding since it requires constant availability of the tracer and requires two examinations at different points in time. The tracer has to be injected prior to revascularisation to measure the area 
at risk and 1 month after revascularisation to measure the final infarct size. ${ }^{3}$

MRI has been investigated as an alternative method to quantify salvaged myocardium that is logistically easier to perform, involves no radiation exposure, can be performed in a single examination ${ }^{3}$ and enables a unique integration of myocardial pathology and measures of myocardial function, such as myocardial strain. ${ }^{4}$ The widely accepted MRI techniques of T1-weighted late gadolinium enhancement for measuring necrotic tissue and T2-weighted MRI for identifying oedematous tissue ${ }^{5}$ are combined and used to quantify salvaged myocardium based on the assumptions that myocardial necrosis on T1-weighted late gadolinium enhancement MRI can be used to delineate the final infarct size, and that myocardial oedema on T2-weighted MRI can be used to delineate the previously ischaemic area at risk. ${ }^{6}$ However, the latter assumption has been a subject of controversial discussion. ${ }^{78}$ While the results of some studies suggest that the area at risk can be delineated by measuring myocardial oedema on T2-weighted MRI, ${ }^{9-13}$ other studies contradict these findings. ${ }^{14-19}$ A panel of experts in the field of postmyocardial infarction MRI recently concluded that oedema on MRI after myocardial infarction should be seen as manifestation of myocardial injury induced by ischemia and reperfusion rather than the previously ischaemic area at risk. ${ }^{20}$

The purpose of this meta-regression analysis was to summarise existing data on the relation between the time from symptom onset until revascularisation (time to reperfusion) and the myocardial salvage index (MSI) calculated as proportion of non-necrotic myocardium inside oedematous myocardium on T2-weighted and T1-weighted late gadolinium enhancement MRI after ST-segment elevation myocardial infarction (STEMI).

\section{MATERIALS AND METHODS}

Reporting of this systematic review and meta-regression analysis followed the Preferred Reporting Items for Systematic reviews and Meta-Analyses (PRISMA) guidelines. $^{21}$

\section{Inclusion and exclusion criteria, search strategy and data extraction}

Included studies had to fulfil the following inclusion criteria: (1) STEMI in all study patients; (2) primary percutaneous intervention as part of emergency care; (3) reporting of the MSI calculated as proportion of nonnecrotic myocardium inside oedematous myocardium on T2-weighted and T1-weighted late gadolinium enhancement MRI in the first week after STEMI or alternatively reporting of the spatial extent of oedematous left ventricular myocardium on T2-weighted MRI along with the spatial extent of left ventricular necrotic myocardium on T1-weighted late gadolinium enhancement MRI, which enabled calculation of the MSI; (4) application of a unit compatible to the percentage of the whole left ventricular myocardium for measuring oedema and necrosis on MRI (see online supplemental table 1); (5) reporting of CI, SD or IQR for the MSI or the spatial extents of myocardial oedema and necrosis on T2-weighted and T1-weighted late gadolinium enhancement MRI; (6) reporting of the time to reperfusion and (7) publication in English, German or French. Animal studies were excluded.

MEDLINE, EMBASE and ISI Web of Science were searched from inception until 16 May 2020. The search term for every database can be found in online supplemental file 1. Titles and abstracts of references found in the databases were screened before a full-text review was performed. The bibliography of included studies and reviews was screened for further eligible studies.

General information about the studies were extracted onto a predefined datasheet along with information on the time to reperfusion, MRI results, used MRI technique and basic patient characteristics.

The eligibility criteria and the search term were set up by two reviewers (BK and MD) and adjusted by discussion in the research group on non-invasive cardiovascular imaging at Charité-Universitätsmedizin Berlin. BK and HS performed the database search, title and abstract review, full-text review and data extraction independently; discrepancies were resolved by discussion, if necessary together with MD.

\section{Statistical analysis}

If included studies did not state the MSI but the spatial extents of oedematous and necrotic myocardium measured by T2-weighted and T1-weighted late gadolinium enhanced MRI, we calculated the MSI ourselves as the proportion of non-necrotic myocardium inside oedematous myocardium on T2-weighted and T1-weighted late gadolinium enhancement MRI. The Delta Method was applied to estimate the variance andSD. ${ }^{22}$

To get an overview of the data, random effects models were applied to calculate pooled values for all extracted continuous variables: the MSI on T2-weighted and T1-weighted late gadolinium enhancement MRI, time to reperfusion, mean age and percent of male patients. The results of sub-groups within studies were included separately when available. We did not consider these observations to be independent from each other because of similar study methods. The intercept was thus allowed to vary randomly for each study to account for multiple observations per study. Each patient group's result was weighted by the inverse of its squared estimated SE. In case of the percent of male patients, the logit transformation was used to stabilise the variance and avoid a variance-on-mean relationship. ${ }^{23}$ Furthermore, we calculated frequency distributions for all extracted categorical variables: T2-weighted MRI sequence, T1-weighted late gadolinium enhancement MRI sequence, T2-weighted MRI interpretation method, T1-weighted late gadolinium enhancement MRI interpretation method and timing of MRI. The individual categories for each categorical variable can be found in table 1. Myocardial oedema appears 
Table 1 Basic characteristics of included study populations and MRI technique used by included studies

\section{Basic characteristics of included study populations}

\begin{tabular}{|c|c|}
\hline Characteristic & Pooled mean $(95 \% \mathrm{Cl})$ \\
\hline Age, years & 59.9 (95\% Cl: 59.0 to 60.7$)$ \\
\hline Male, $\%$ of patients & 81.7 (95\% Cl: 79.3 to 83.9$)$ \\
\hline \multicolumn{2}{|l|}{ MRI technique used by included studies } \\
\hline Technical parameter & $\%$ of included studies (n) \\
\hline \multicolumn{2}{|l|}{ Timing of MRI } \\
\hline 3-7 days after STEMI & $79 \%(30)$ \\
\hline 1-2 days after STEMI & $21 \%(8)$ \\
\hline \multicolumn{2}{|l|}{ T2-weighted MRI sequence* } \\
\hline T2-weighted dark-blood TSE/FSE with IR (STIR) & $90 \%(34)$ \\
\hline T2-prepared bright-blood single-shot balanced SSFP & $11 \%(4)$ \\
\hline Hybrid TSE-SSFP (ACUTE) & $5 \%(2)$ \\
\hline BLADE k-space coverage for dark-blood TSE & $3 \%(1)$ \\
\hline \multicolumn{2}{|l|}{ T1-weighted late gadolinium enhancement MRI sequence } \\
\hline PSIR using segmented FLASH readout (SPGR) & $97 \%(37)$ \\
\hline IR with single-shot SSFP & $3 \%(1)$ \\
\hline \multicolumn{2}{|l|}{ T2-weighted MRI interpretation } \\
\hline Signal intensity $>2$ SD above remote myocardium & $66 \%(25)$ \\
\hline Manual contouring & $29 \%(11)$ \\
\hline FWHM algorithm & $5 \%(2)$ \\
\hline \multicolumn{2}{|c|}{ T1-weighted late gadolinium enhancement MRI interpretation $†$} \\
\hline Signal intensity $>5 \mathrm{SD}$ above remote myocardium & $37 \%(14)$ \\
\hline Signal intensity $>2$ SD above remote myocardium & $16 \%(6)$ \\
\hline Signal intensity $>3 S D$ above remote myocardium & $3 \%(1)$ \\
\hline Manual contouring & $29 \%(11)$ \\
\hline FWHM algorithm & $11 \%(4)$ \\
\hline Heiberg's method & $8 \%(3)$ \\
\hline
\end{tabular}

*One included study compared four T2-weighted MRI sequences.

†One included study applied two T1-weighted late gadolinium enhancement MRI interpretation methods.

ACUTE, acquisition for cardiac unified T2 oedema; FLASH, fastlow angle shot; FSE, fast spin echo; FWHM, full width at half maximum; IR, inversion recovery; PSIR, phase-sensitive inversion recovery; SPGR, spoiled gradient echo; SSFP, steady-state free precession; STEMI, ST-segment elevation myocardial infarction; STIR, short-tau inversion recovery; TSE, turbo spin echo.

to be more stable in the time window between days 3 and 7 after STEMI, ${ }^{20}$ and we thus categorised the timing of MRI into 1-2 days and 3-7 days after STEMI.

A mixed effects model was used to test for an association between the time to reperfusion and the MSI. The MSI was used as a dependent variable. The time to reperfusion was used as fixed effect. Again, the intercept was allowed to vary randomly for each study to account for multiple observations per study. Between-study heterogeneity was evaluated using Cochran's $Q$ Test. We weighted each patient group's result by the inverse of the squared estimated SE of the mean of the MSI. On request of the reviewers, the model was also calculated after excluding outlying patient groups having a mean time to perfusion of less than 2 hours.

To explore the between-study heterogeneity in the mixed effects model, basic patient characteristics and MRI parameters were included in the mixed effects model with the time to reperfusion as fixed effect and study as random effect. The mean age and gender distribution were included as continuous fixed effects, while the timing of MRI, T2-weighted MRI sequence, T1-weighted late gadolinium enhancement MRI sequence, T2-weighted MRI interpretation method and T1-weighted late gadolinium enhancement MRI interpretation method were included as categorical fixed effects. Each of the variables was included separately in the mixed effects model. The likelihood ratio test was applied to test whether the included variable provided a significantly better fit. All significant confounding variables were included together in the mixed effects model. Interactions were not included to avoid overfitting. The unexplained between-study variance $\sigma^{2}$ was compared between the hereby resulting model and the original model without inclusion of confounding variables. 
We assumed statistical significance for $\mathrm{p}$ values of less than 0.05. We used R (V.3.6.0, 2019, R Foundation of Statistical Computing) for the whole statistical analysis. The metafor R package was used to set up random and mixed effects models. ${ }^{24}$ Two reviewers (PS and BK) planned the statistical analysis, which was performed afterwards by BK. The results were regularly discussed with and checked by PS to assure accuracy. The final results were discussed in the research group on non-invasive cardiovascular imaging at Charité-Universitätsmedizin Berlin.

\section{Risk of bias assessment}

We applied the component approach ${ }^{25}$ for assessing the risk of bias in individual studies, and thus developed a set of items for the domains of bias we considered most relevant to this meta-analysis: selection bias, attrition bias and detection bias. The items are explained in detail in online supplemental table 2 . The risk of publication bias was assessed across studies by searching for obvious asymmetry in a funnel plot created with the data on the MSI. Begg and Mazumdar's correlation test ${ }^{26}$ and Egger et als regression test ${ }^{27}$ were applied as additional tests for publication bias.

\section{Patient and public involvement}

No patients were involved in setting the research question or design of the study. No patients were asked to advise on the interpretation of the results. We aim to present the results of the study to a wide audience, including patients, health professionals and members of the public.

\section{RESULTS}

Study selection is summarised by the PRISMA flow chart in figure 1. We included 38 studies with 5106 patients in this meta-regression analysis. ${ }^{28-61}$ Eighteen studies calculated and reported the MSI as proportion of nonnecrotic myocardium inside oedematous myocardium on T2-weighted and T1-weighted late gadolinium enhancement MRI. For 19 studies, we calculated the MSI using available data on the spatial extent of oedematous and necrotic left ventricular myocardium on T2-weighted and T1-weighted late gadolinium enhancement MRI. Table 1 summarises basic patient characteristics and the used MRI technique.

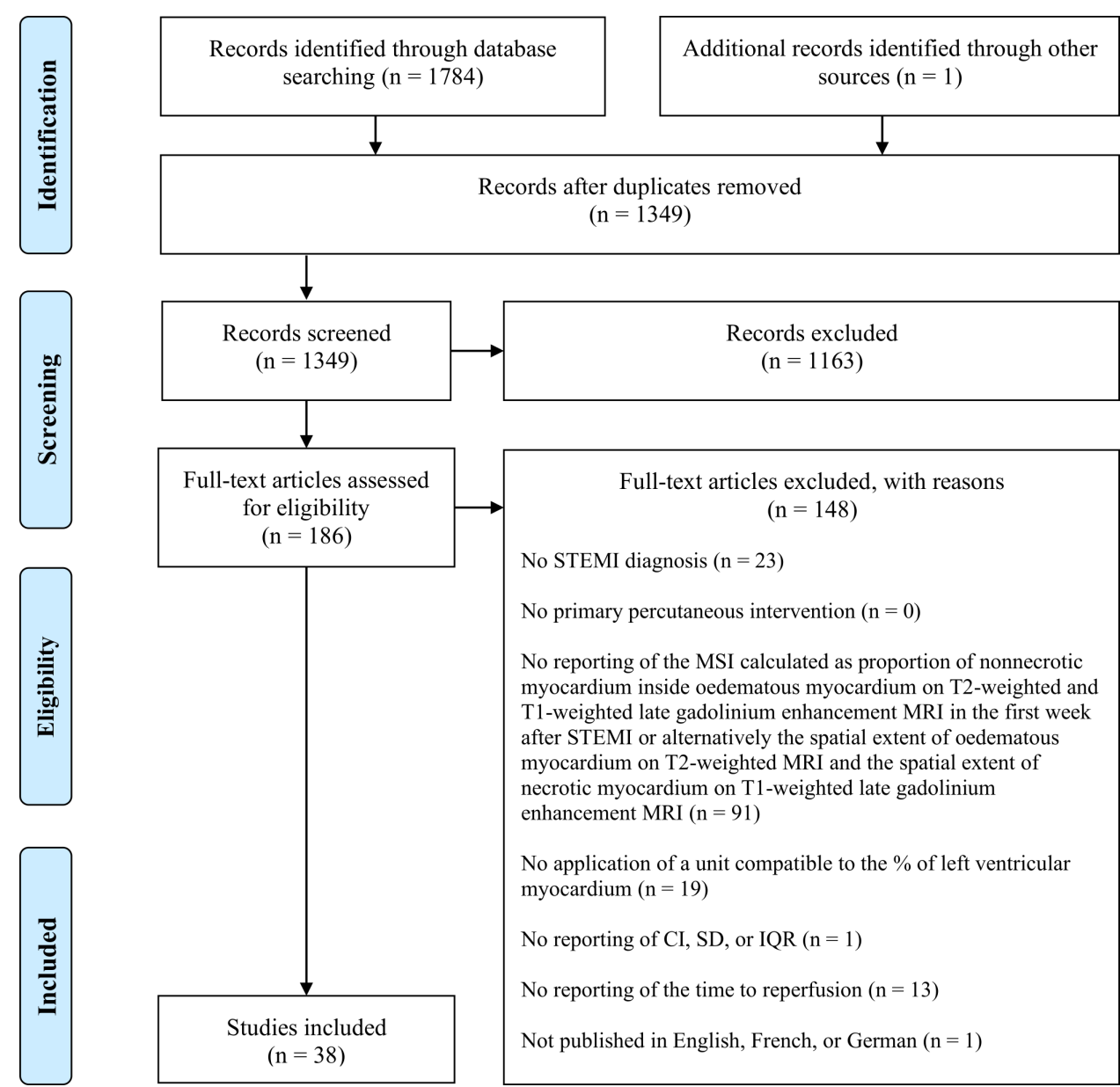

Figure 1 PRISMA flow chart; abstract and title review was performed of 1784 references that were found in MEDLINE, EMBASE and ISI Web of Science. We performed full-text review of 186 studies and included 38 studies in this meta-regression analysis. MSI, myocardial salvage index; PRISMA, Preferred Reporting Items for Systematic reviews and Meta-Analyses; STEMI, ST-segment elevation myocardial infarction; time to reperfusion, time from symptom onset until revascularisation. 


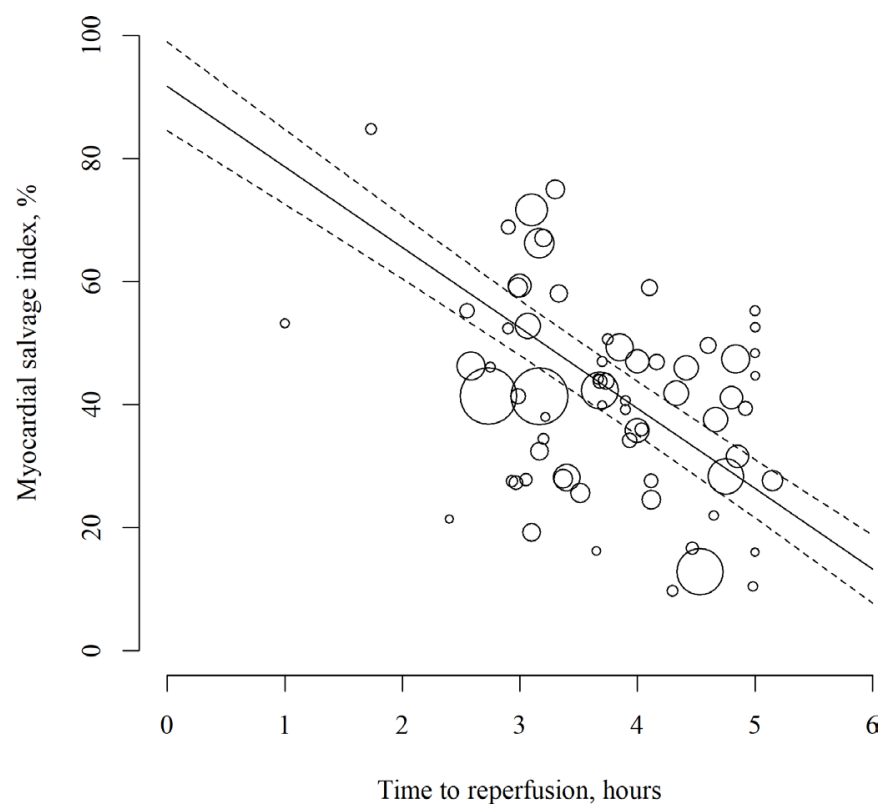

Figure 2 Inverse correlation between the time to reperfusion and the myocardial salvage index. The dashed lines represent the lower and upper $95 \% \mathrm{Cl}$. The point size reflects the weight of a patient group, which was calculated as the inverse of the squared estimated SE. Time to reperfusion, time from symptom onset until revascularisation.

The pooled MSI was $42.6 \%$ (95\% CI: 38.9 to 47.0 ). The pooled time to reperfusion was $3.8(95 \%$ CI: 3.5 to 4.0). There was an inverse correlation between the time to reperfusion and the MSI (figure 2). Every hour of delay in reperfusion was associated with an absolute decrease of $13.1 \%$ (95\% CI: 11.5 to $14.6 ; \mathrm{p}<0.001)$ in the MSI. Between-study heterogeneity was considerable $\left(\sigma^{2}=167.8\right)$. Details of the mixed effects model can be found in table 2. The results of the mixed effects model calculated after excluding two outlying patient groups with a mean time to perfusion of less than 2 hours are summarised in online supplemental table 3.

Including basic patient characteristics and MRI parameters separately in the mixed effects model revealed that the inclusion of the timing of MRI $\left(\chi^{2}(1)=11.5, p<0.001\right)$, T2-weighted MRI interpretation method $\left(\chi^{2}(2)=7.8\right.$, $\mathrm{p}=0.020)$, T1-weighted late gadolinium enhancement MRI interpretation method $\left(\chi^{2}(5)=15.3, \mathrm{p}=0.009\right)$ and gender distribution $\left(\chi^{2}(1)=11.3, p<0.001\right)$ resulted in a significantly better fit of the mixed effects model, while the inclusion of the T2-weighted MRI sequence $\left(\chi^{2}(3)=5.6, \quad \mathrm{p}=0.131\right)$, T1-weighted late gadolinium enhancement MRI sequence $\left(\chi^{2}(1)=0.5, \mathrm{p}=0.468\right)$ and mean age $\left(\chi^{2}(1)<0.1, p=0.995\right)$ did not. Inclusion of all significant confounding variables in the mixed effects model reduced the between-study heterogeneity by $45.2 \%$ to $\sigma^{2}=91.9$. The detailed parameters of this model can be found in online supplemental table 4 .

The risk of selection bias, attrition bias and detection bias in included studies is summarised in figure 3. The detailed judgements for every study can be found in online supplemental tables 5-7. We neither find evidence
Table 2 Model parameters of the mixed effects model

\begin{tabular}{|c|c|c|c|c|}
\hline \multicolumn{5}{|l|}{ Model fit } \\
\hline logLik & AIC & \multicolumn{3}{|l|}{ BIC } \\
\hline-432.8 & 871.6 & \multicolumn{3}{|l|}{878.3} \\
\hline \multicolumn{5}{|l|}{ Random effects } \\
\hline \multirow[t]{2}{*}{ Factor } & \multirow[t]{2}{*}{$\sigma 2$} & \multirow[t]{2}{*}{$\sigma$} & \multirow{2}{*}{\multicolumn{2}{|c|}{$\begin{array}{l}\text { Cochran's Q Test } \\
\text { for heterogeneity } \\
\text { P value }\end{array}$}} \\
\hline & & & & \\
\hline Study & $167.8^{*}$ & $13.0^{*}$ & $<0.001$ & \\
\hline \multicolumn{5}{|l|}{ Fixed effects } \\
\hline Factor & Estimate & $P$ value & $\begin{array}{l}\text { Lower } \\
95 \% \mathrm{Cl}\end{array}$ & $\begin{array}{l}\text { Upper } \\
95 \% \mathrm{Cl}\end{array}$ \\
\hline (intercept) & 91.7 & $<0.001$ & 84.5 & 98.9 \\
\hline $\begin{array}{l}\text { Time to reperfusion, } \\
\text { hours }\end{array}$ & -13.1 & $<0.001$ & -14.6 & -11.5 \\
\hline
\end{tabular}

*Exploration of heterogeneity: Separate inclusion of the timing of MRI $\left(\chi^{2}(1)=11.5, p<0.001\right)$, T2-weighted MRI interpretation method $\left(\chi^{2}(2)=7.8, p=0.020\right)$, T1-weighted late gadolinium enhancement MRI interpretation method $\left(\chi^{2}(5)=15.3, p=0.009\right)$ and gender distribution $\left(\chi^{2}(1)=11.3, p<0.001\right)$ resulted in a significantly better fit of the mixed effects model, while the inclusion of the T2-weighted MRI sequence $\left(\chi^{2}(3)=5.6, p=0.131\right)$, T1-weighted late gadolinium enhancement MRI sequence $\left(\chi^{2}(1)=0.5, p=0.468\right)$ and mean age $\left(\chi^{2}(1)<0.1, p=0.995\right)$ did not. Inclusion of all significant confounding variables reduced the between-study heterogeneity by $45.2 \%$ to $\sigma^{2}=91.9$ (see online supplemental table 4).

AIC, Akaike information criterion; BIC, Bayesian information criterion; logLik, log-likelihood.

of publication bias across studies by searching for obvious asymmetry in the created funnel plot with the data on the MSI measured by MRI (see online supplemental figure 1) nor by applying Begg and Mazumdar's rank correlation test $(\mathrm{z}=-1.4, \mathrm{p}=0.173)$ and Egger et al $\mathrm{s}$ regression test $(\mathrm{t}=0.7, \mathrm{p}=0.507)$.

All raw data extracted from included studies can be found in online supplemental table 8 .

\section{DISCUSSION}

This meta-regression analysis was conducted to summarise existing data on the relation between the time to reperfusion and the MSI on T2-weighted and T1-weighted late gadolinium enhancement MRI after STEMI. The analysis showed that a short delay between symptom onset and revascularisation is associated with a large MSI, while a long delay between symptom onset and revascularisation is associated with a small MSI. There is considerable heterogeneity between studies.

\section{Comparison with other studies}

We did not find a meta-analysis in MEDLINE, EMBASE, ISI Web of Science, Cochrane or PROSPERO that summarised data on the relation between the time to reperfusion and the MSI on T2-weighted and T1-weighted late gadolinium enhancement MRI after STEMI. While 


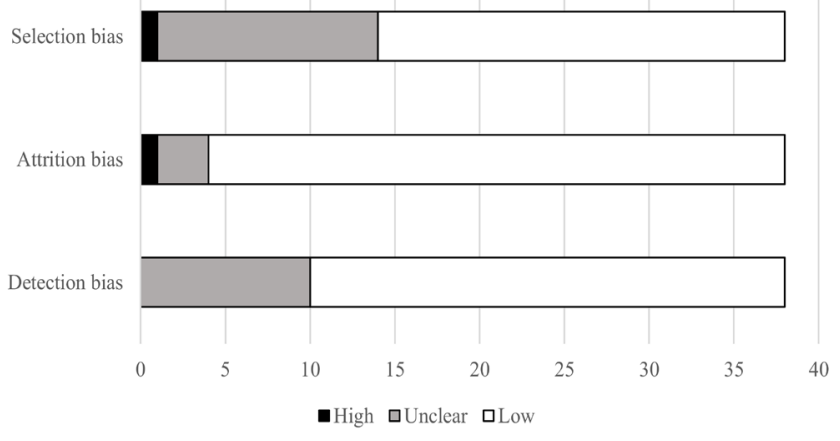

Figure 3 Risk of bias in individual studies. The risk of selection bias was judged as low in 25 of 34 studies, attrition bias in 34 studies and detection bias in 28 studies. In one study, the risk of selection bias was judged as high, and in another study, the risk of attrition bias.

most of, in this meta-regression analysis, included studies did not primarily study the impact of revascularisation delay on the MSI measured by T2-weighted and T1-weighted late gadolinium enhancement MRI, Francone et al subcategorised study patients into four time to reperfusion intervals. ${ }^{40}$ Consistent with the main result of this meta-regression analysis, the data of Francone et al show an inverse correlation between the time to reperfusion and the MSI on T2-weighted and T1-weighted late gadolinium enhancement MRI.

As mentioned in the Introduction section, opinions differ on whether measurement of the extent of myocardial oedema by T2-weighted MRI allows delineation of the previously ischaemic area at risk differ. ${ }^{7-19}$ In their landmark studies of the wavefront phenomenon of myocardial death, Reimer et al showed that necrotic myocardium gradually expands from the subendocardium toward the subepicardium in the ischaemic area at risk as long as ischemia persists, while this expansion can be halted by revascularisation of the ischaemic area. ${ }^{62} 63$ The gradual expansion of myocardial necrosis measured by T1-weighted late gadolinium enhancement MRI within infarction-induced myocardial oedema measured by T2-weighted MRI with increasing time to reperfusion showed in this meta-analysis suggests a connection between the area at risk and the extent of myocardial oedema. However, the data cannot exclude underestimation or overestimation of the area at risk using myocardial oedema on T2-weighted MRI, and therefore the conclusion that myocardial oedema on T2-weighted MRI delineates the area at risk cannot be made.

\section{Meaning of the study and future perspective}

Regardless of if myocardial oedema on T2-weighted MRI delineates the area at risk, underestimates or overestimates it, the gradual expansion of necrosis within infarctioninduced oedema with increasing time to reperfusion indicates that measurement of the proportion of oedematous myocardium without necrosis by T2-weighted and T1-weighted late gadolinium enhancement MRI might be of use for evaluating therapeutic efficiency, which may be helpful for studies investigating cardioprotective strategies.

The heterogeneity between studies suggests that other factors different from the time to reperfusion affect the proportion of oedematous myocardium without necrosis measured by T2-weighted and T1-weighted late gadolinium enhancement MRI after myocardial infarction. Since the heterogeneity analysis revealed significant confounding effects for the timing of imaging and image interpretation, differences in MRI methodologies should be resolved in the future to improve comparability of the results. The need for a standardisation of postmyocardial infarction MRI methodologies has also recently been highlighted by experts in the field. ${ }^{20}$ The use of T2-mapping and T1-mapping MRI, which is a valuable and increasingly used alternative to conventional T2-weighted and T1-weighted late gadolinium enhancement MRI, may be a step forward in this regard since it allows a less subjective and more consistent delineation of oedematous and fibrotic myocardium. ${ }^{64}$ The heterogeneity analysis furthermore suggests that gender differences should be considered when interpreting the MSI, which has previously been described as well. ${ }^{65} 66$ Differences in cardioprotective strategies apart from the time to reperfusion may explain a fraction of the remaining heterogeneity. ${ }^{43}$ Further research on the factors influencing the MSI measured by MRI is needed before a reliable evaluation of therapy efficiency may be possible by measuring the MSI with MRI.

\section{Strengths and limitations}

This systematic review and meta-regression analysis has methodological strengths. Reporting followed the PRISMA guidelines. ${ }^{21}$ After a comprehensive search in three electronic databases, a large number of studies could be included. State-of-the-art statistical tests for metaregression analyses were applied for data analysis. We did not register a review protocol a priori, which increased the likelihood of our post hoc decisions to be biassed. ${ }^{21}$ Last, data on T2-mapping and T1-mapping MRI are not included in this meta-regression analysis. As mentioned above, mapping MRI may overcome inconsistencies when delineating the extent of myocardial oedema and fibrosis; however, only a few studies already applied mapping MRI for measuring the MSI. To have enough data for a conclusive meta-regression analysis, we therefore decided to search and include studies that used conventional T2-weighted and T1-weighted late gadolinium enhancement MRI.

\section{CONCLUSIONS}

The MSI calculated as proportion of non-necrotic myocardium inside oedematous myocardium on T2-weighted and T1-weighted late gadolinium enhancement MRI after revascularisation in STEMI correlates inversely with the time to reperfusion, which indicates that cardioprotection 
achieved by minimising the time to reperfusion leads to a higher MSI measured by MRI. The analysis revealed considerable heterogeneity between studies. A substantial part of the heterogeneity could be explained by differences in the gender distribution, timing and interpretation of MRI suggesting that the MRI-assessed MSI is not only influenced by cardioprotective therapy but also by patient characteristics and MRI parameters. One reviewer performed the database search, data extraction and statistical analysis, which limits the results.

Contributors Conceptualisation: BK and MD. Methodology: BK. Data curation: BK and HS. Formal analysis: BK and PS. Supervision: MD and PS. Visualisation: BK. Writing —original draft: BK. Writing — review and editing: MD. Guarantors: BK and MD.

Funding We acknowledge support from the German Research Foundation (DFG), the German Federal Ministry of Education and Research (BMBF) and the Open Access Publication Fund of Charité-Universitätsmedizin Berlin. The funders had no role in the study design; in the data collection, analysis and interpretation of data; in the writing of the report and in the decision to submit the article for publication. The researches were independent from funders. All authors had full access to all of the data (including statistical reports and tables) in the study and can take responsibility for the integrity of the data and the accuracy of the data analysis.

Competing interests MD and PS received financial support from the German Research Foundation (DFG) and the German Federal Ministry of Education and Research (BMBF) for the submitted work; outside the submitted work, MD received grants from the German Foundation of Heart Research, GE Healthcare, Bracco, Guerbet, Toshiba Medical Systems, Siemens Medical Solutions, Philips Medical Systems, German Research Foundation (DFG) and the European Union (funding programme FP7), and personal fees from German Research Foundation (DFG), Guerbet, Cardiac MR Academy Berlin, Bayer-Schering, Toshiba Medical Systems and Springer; outside the submitted work, PS received grants from the German Research Foundation (DFG), European Union and Bayer Pharma AG; no other relationships or activities that could appear to have influenced the submitted work.

Patient consent for publication Not required.

Provenance and peer review Not commissioned; externally peer reviewed.

Data availability statement All data relevant to the study are included in the article or uploaded as supplemental information.

Open access This is an open access article distributed in accordance with the Creative Commons Attribution Non Commercial (CC BY-NC 4.0) license, which permits others to distribute, remix, adapt, build upon this work non-commercially, and license their derivative works on different terms, provided the original work is properly cited, appropriate credit is given, any changes made indicated, and the use is non-commercial. See: http://creativecommons.org/licenses/by-nc/4.0/.

\section{ORCID iD}

Benjamin Kendziora http://orcid.org/0000-0002-5234-5380

\section{REFERENCES}

1 WHO. Global status report on noncommunicable diseases 2014. Geneva: World Health Organization, 2014.

2 Fox KAA, Steg PG, Eagle KA, et al. Decline in rates of death and heart failure in acute coronary syndromes, 1999-2006. JAMA 2007;297:1892-900.

3 Bøtker HE, Kaltoft AK, Pedersen SF, et al. Measuring myocardial salvage. Cardiovasc Res 2012;94:266-75.

4 Mangion K, McComb C, Auger DA, et al. Magnetic resonance imaging of myocardial strain after acute ST-segment-elevation myocardial infarction: a systematic review. Circ Cardiovasc Imaging 2017;10:e006498.

5 American College of Cardiology Foundation Task Force on Expert Consensus Documents, Hundley WG, Bluemke DA, et al. ACCF/ ACR/AHA/NASCI/SCMR 2010 expert consensus document on cardiovascular magnetic resonance: a report of the American College of cardiology Foundation Task force on expert consensus documents. J Am Coll Cardiol 2010;55:2614-62.
6 Rochitte CE, Azevedo CF. The myocardial area at risk. Heart 2012;98:348-50.

7 Arai AE, Leung S, Kellman P. Controversies in cardiovascular MR imaging: reasons why imaging myocardial T2 has clinical and pathophysiologic value in acute myocardial infarction. Radiology 2012;265:23-32.

8 Croisille P, Kim HW, Kim RJ. Controversies in cardiovascular MR imaging: T2-weighted imaging should not be used to delineate the area at risk in ischemic myocardial injury. Radiology 2012;265:12-22.

9 Aletras AH, Tilak GS, Natanzon A, et al. Retrospective determination of the area at risk for reperfused acute myocardial infarction with T2weighted cardiac magnetic resonance imaging: histopathological and displacement encoding with stimulated echoes (DENSE) functional validations. Circulation 2006;113:1865-70.

10 Berry C, Kellman P, Mancini C, et al. Magnetic resonance imaging delineates the ischemic area at risk and myocardial salvage in patients with acute myocardial infarction. Circ Cardiovasc Imaging 2010;3:527-35.

11 Carlsson M, Ubachs JFA, Hedström E, et al. Myocardium at risk after acute infarction in humans on cardiac magnetic resonance: quantitative assessment during follow-up and validation with singlephoton emission computed tomography. JACC Cardiovasc Imaging 2009;2:569-76.

12 García-Dorado D, Oliveras J, Gili J, et al. Analysis of myocardial oedema by magnetic resonance imaging early after coronary artery occlusion with or without reperfusion. Cardiovasc Res 1993;27:1462-9.

13 Tilak GS, Hsu L-Y, Hoyt RF, et al. In vivo T2-weighted magnetic resonance imaging can accurately determine the ischemic area at risk for 2-day-old nonreperfused myocardial infarction. Invest Radiol 2008;43:7-15.

14 Johnston DL, Brady TJ, Ratner AV, et al. Assessment of myocardial ischemia with proton magnetic resonance: effects of a three hour coronary occlusion with and without reperfusion. Circulation 1985;71:595-601.

15 Kim RJ, Fieno DS, Parrish TB, et al. Relationship of MRI delayed contrast enhancement to irreversible injury, infarct age, and contractile function. Circulation 1999;100:1992-2002.

16 Mewton N, Rapacchi S, Augeul L, et al. Determination of the myocardial area at risk with pre- versus post-reperfusion imaging techniques in the pig model. Basic Res Cardiol 2011:106:1247-57.

17 Miller DD, Johnston DL, Dragotakes D, et al. Effect of hyperosmotic mannitol on magnetic resonance relaxation parameters in reperfused canine myocardial infarction. Magn Reson Imaging 1989;7:79-88.

18 Ryan T, Tarver RD, Duerk JL, et al. Distinguishing viable from infarcted myocardium after experimental ischemia and reperfusion by using nuclear magnetic resonance imaging. J Am Coll Cardiol 1990;15:1355-64.

19 Thuny F, Lairez O, Roubille F, et al. Post-conditioning reduces infarct size and edema in patients with ST-segment elevation myocardial infarction. J Am Coll Cardiol 2012;59:2175-81.

20 Ibanez B, Aletras AH, Arai AE, et al. Cardiac MRI endpoints in myocardial infarction experimental and clinical trials. J Am Coll Cardiol 2019;74:238-56

21 Moher D, Liberati A, Tetzlaff J, et al. Preferred reporting items for systematic reviews and meta-analyses: the PRISMA statement. Ann Intern Med 2009;151:264-9.

22 van der Vaart AW, Wellner JA. The delta-method. In: Weak convergence and empirical processes: with applications to statistics. New York, NY: Springer, 1996: 372-400.

23 Wang N. How to conduct a meta-analysis of proportions in R: a comprehensive tutorial, 2018.

24 Viechtbauer W. Conducting meta-analyses in R with the metafor package. J Stat Soft 2010;1:2010.

25 Mueller M, D’Addario M, Egger M, et al. Methods to systematically review and meta-analyse observational studies: a systematic scoping review of recommendations. BMC Med Res Methodol 2018;18:44.

26 Begg CB, Mazumdar M. Operating characteristics of a RANK correlation test for publication bias. Biometrics 1994;50:1088-101.

27 Egger M, Davey Smith G, Schneider M, et al. Bias in meta-analysis detected by a simple, graphical test. BMJ 1997;315:629-34.

28 Alkhalil M, Borlotti A, De Maria GL, et al. Dynamic changes in injured myocardium, very early after acute myocardial infarction, quantified using T1 mapping cardiovascular magnetic resonance. J Cardiovasc Magn Reson 2018;20:82.

29 Bin Gwag H, Park TK, Song YB, et al. Morphine does not affect myocardial salvage in ST-segment elevation myocardial infarction. PLoS One 2017;12.

30 Borlotti A, Jerosch-Herold M, Liu D, et al. Acute microvascular impairment post-reperfused STEMI is reversible and has additional 
clinical predictive value: a CMR OxAMI study. JACC CardiovasC Imaging 2019;12:1783-93.

31 Cameron D, Siddiqi N, Neil CJ, et al. T mapping for assessment of myocardial injury and microvascular obstruction at one week post myocardial infarction. Eur J Radiol 2015.

32 Chung S, Song YB, Hahn J-Y, et al. Impact of white blood cell count on myocardial salvage, infarct size, and clinical outcomes in patients undergoing primary percutaneous coronary intervention for ST-segment elevation myocardial infarction: a magnetic resonance imaging study. Int J Cardiovasc Imaging 2014;30:129-36.

33 Crimi G, Pica S, Raineri C, et al. Remote ischemic post-conditioning of the lower limb during primary percutaneous coronary intervention safely reduces enzymatic infarct size in anterior myocardial infarction: a randomized controlled trial. JACC Cardiovasc Interv 2013:6:1055-63.

34 de Waha S, Eitel I, Desch S, et al. Prognosis after ST-elevation myocardial infarction: a study on cardiac magnetic resonance imaging versus clinical routine. Trials 2014;15:249.

35 Desch S, Engelhardt H, Meissner J, et al. Reliability of myocardial salvage assessment by cardiac magnetic resonance imaging in acute reperfused myocardial infarction. Int $J$ Cardiovasc Imaging 2012;28:263-72.

36 Eitel I, Desch S, de Waha S, et al. Long-term prognostic value of myocardial salvage assessed by cardiovascular magnetic resonance in acute reperfused myocardial infarction. Heart 2011;97:2038-45.

37 Eitel I, Kubusch K, Strohm O, et al. Prognostic value and determinants of a hypointense infarct core in T2-weighted cardiac magnetic resonance in acute reperfused ST-elevation-myocardial infarction. Circ Cardiovasc Imaging 2011;4:354-62.

38 Eitel I, Wöhrle J, Suenkel H, et al. Intracoronary compared with intravenous bolus abciximab application during primary percutaneous coronary intervention in ST-segment elevation myocardial infarction: cardiac magnetic resonance substudy of the AIDA STEMI trial. J Am Coll Cardiol 2013;61:1447-54.

39 Engblom H, Strauss DG, Heden B, et al. The evaluation of an electrocardiographic myocardial ischemia acuteness score to predict the amount of myocardial salvage achieved by early percutaneous coronary intervention clinical validation with myocardial perfusion single photon emission computed tomography and cardiac magnetic resonance. J Electrocardiol 2011;44:525-32.

40 Francone M, Bucciarelli-Ducci C, Carbone I, et al. Impact of primary coronary angioplasty delay on myocardial salvage, infarct size, and microvascular damage in patients with ST-segment elevation myocardial infarction: insight from cardiovascular magnetic resonance. J Am Coll Cardiol 2009;54:2145-53.

41 Fuernau G, Eitel I, Franke V, et al. Myocardium at risk in ST-segment elevation myocardial infarction comparison of T2-weighted edema imaging with the MR-assessed endocardial surface area and validation against angiographic scoring. JACC Cardiovasc Imaging 2011:4:967-76.

42 Garg P, Broadbent DA, Swoboda PP, et al. Acute infarct extracellular volume mapping to quantify myocardial area at risk and chronic infarct size on cardiovascular magnetic resonance imaging. Circ Cardiovasc Imaging 2017;10.

43 Götberg M, Olivecrona GK, Koul S, et al. A pilot study of rapid cooling by cold saline and endovascular cooling before reperfusion in patients with ST-elevation myocardial infarction. Circ Cardiovasc Interv 2010;3:400-7.

44 Grothoff M, Elpert C, Hoffmann J, et al. Right ventricular injury in ST-elevation myocardial infarction: risk stratification by visualization of wall motion, edema, and delayed-enhancement cardiac magnetic resonance. Circ Cardiovasc Imaging 2012;5:60-8.

45 Hadamitzky M, Langhans B, Hausleiter J, et al. The assessment of area at risk and myocardial salvage after coronary revascularization in acute myocardial infarction: comparison between CMR and SPECT. JACC Cardiovasc Imaging 2013;6:358-69.

46 Husser O, Monmeneu JV, Sanchis J, et al. Cardiovascular magnetic resonance-derived intramyocardial hemorrhage after STEMI: influence on long-term prognosis, adverse left ventricular remodeling and relationship with microvascular obstruction. Int J Cardiol 2013;167:2047-54

47 Lee KH, Choi SI, Chun EJ, et al. Aborted myocardial infarction: evaluation of changes in area at risk, late gadolinium enhancement, and perfusion over time and comparison with overt myocardial infarction. AJR Am J Roentgenol 2012;199:328-35.

48 Liu D, Borlotti A, Viliani D, et al. CMR native T1 mapping allows differentiation of reversible versus irreversible myocardial damage in
ST-segment-elevation myocardial infarction: an OxAMI study (Oxford acute myocardial infarction). Circulation Cardiovascular imaging 2017;10.

49 Mandurino-Mirizzi A, Crimi G, Raineri C, et al. Elevated serum uric acid affects myocardial reperfusion and infarct size in patients with ST-segment elevation myocardial infarction undergoing primary percutaneous coronary intervention. J Cardiovasc Med 2018;19:240-6.

50 Masci PG, Ganame J, Strata E, et al. Myocardial salvage by CMR correlates with LV remodeling and early ST-segment resolution in acute myocardial infarction. JACC Cardiovasc Imaging 2010;3:45-51.

51 Mather AN, Fairbairn TA, Artis NJ, et al. Timing of cardiovascular MR imaging after acute myocardial infarction: effect on estimates of infarct characteristics and prediction of late ventricular remodeling. Radiology 2011;261:116-26.

52 Mather AN, Fairbairn TA, Ball SG, et al. Reperfusion haemorrhage as determined by cardiovascular MRI is a predictor of adverse left ventricular remodelling and markers of late arrhythmic risk. Heart 2011;97:453-9.

53 Monmeneu JV, Bodí V, López-Lereu MP, et al. Analysis of postinfarction salvaged myocardium by cardiac magnetic resonance. predictors and influence on adverse ventricular remodeling. Rev Esp Cardiol 2012;65:634-41.

54 Moral S, Rodríguez-Palomares JF, Descalzo M, et al. Quantification of myocardial area at risk: validation of coronary angiographic scores with cardiovascular magnetic resonance methods. Rev Esp Cardiol 2012:65:1010-7.

55 Nordlund D, Klug G, Heiberg E, et al. Multi-vendor, multicentre comparison of contrast-enhanced SSFP and T2-STIR CMR for determining myocardium at risk in ST-elevation myocardial infarction. Eur Heart J Cardiovasc Imaging 2016;17:744-53.

56 Payne AR, Berry C, Doolin O, et al. Microvascular resistance predicts myocardial salvage and infarct characteristics in ST-elevation myocardial infarction. J Am Heart Assoc 2012;1:e002246.

57 Song YB, Hahn J-Y, Gwon H-C, et al. A high loading dose of clopidogrel reduces myocardial infarct size in patients undergoing primary percutaneous coronary intervention: a magnetic resonance imaging study. Am Heart J 2012;163:500-7.

58 Ubachs JFA, Engblom H, Erlinge D, et al. Cardiovascular magnetic resonance of the myocardium at risk in acute reperfused myocardial infarction: comparison of T2-weighted imaging versus the circumferential endocardial extent of late gadolinium enhancement with transmural projection. J Cardiovasc Magn Reson 2010;12:18.

59 Viallon M, Mewton N, Thuny F, et al. T2-weighted cardiac MR assessment of the myocardial area-at-risk and salvage area in acute reperfused myocardial infarction: comparison of state-of-the-art dark blood and bright blood T2-weighted sequences. J Magn Reson Imaging 2012;35:328-39.

60 Wright J, Adriaenssens T, Dymarkowski S, et al. Quantification of myocardial area at risk with T2-weighted CMR: comparison with contrast-enhanced CMR and coronary angiography. JACC Cardiovasc Imaging 2009;2:825-31.

$61 \mathrm{Xu}$ J, Song YB, Hahn J-Y, et al. Comparison of magnetic resonance imaging findings in non-ST-segment elevation versus ST-segment elevation myocardial infarction patients undergoing early invasive intervention. Int J Cardiovasc Imaging 2012;28:1487-97.

62 Reimer KA, Jennings RB. The "wavefront phenomenon" of myocardial ischemic cell death II. Transmural progression of necrosis within the framework of ischemic bed size (myocardium at risk) and collateral flow. Laboratory investigation. J Tech Method Pathol 1979;40:633-44.

63 Reimer KA, Lowe JE, Rasmussen MM, et al. The wavefront phenomenon of ischemic cell death. 1. myocardial infarct size vs duration of coronary occlusion in dogs. Circulation 1977;56:786-94.

64 Mavrogeni S, Apostolou D, Argyriou P, et al. T1 and T2 mapping in cardiology: "mapping the obscure object of desire". Cardiology 2017;138:207-17.

65 Canali E, Masci P, Bogaert J, et al. Impact of gender differences on myocardial salvage and post-ischaemic left ventricular remodelling after primary coronary angioplasty: new insights from cardiovascular magnetic resonance. Eur Heart J Cardiovasc Imaging 2012;13:948-53.

66 Mehilli J, Ndrepepa G, Kastrati A, et al. Gender and myocardial salvage after reperfusion treatment in acute myocardial infarction. $J$ Am Coll Cardiol 2005;45:828-31. 